\title{
Event Based Data from a 2070 Controller
}

Automated Traffic Signal Performance Measures Workshop

$$
\text { January } 13^{\text {th }}, 2016
$$

Ed Smaglik, Northern Arizona University

Anuj Sharma, lowa State University

Other Contributors:

Sirisha Kothuri, PSU 


\section{Agenda}

- Introduction

- Motivation

- Module Development

- Deployment Sites

- Data Collection

- Preliminary Results

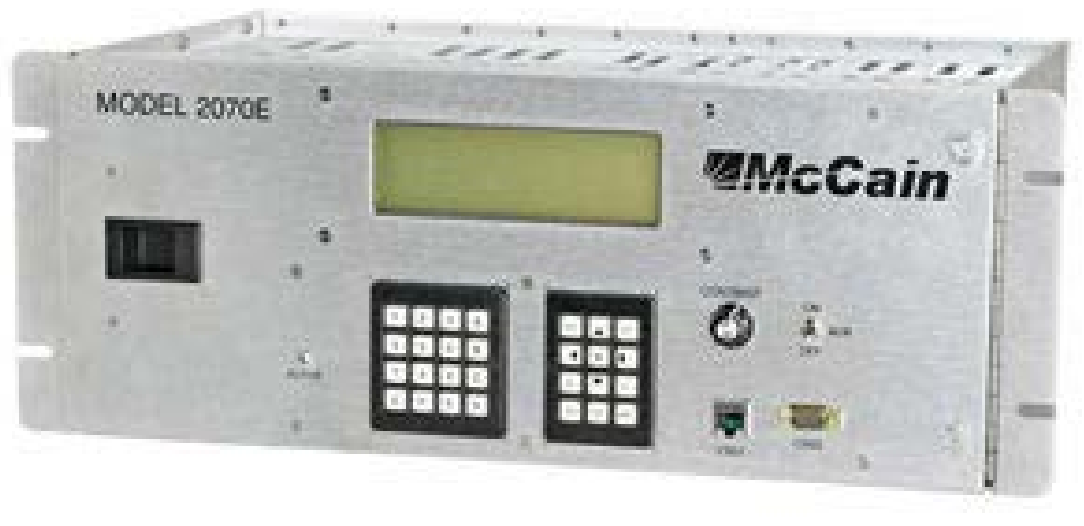

- Next Steps 


\section{SPR 781: Snapshot}

- Funding Agency: Oregon DOT

- Project Title: Improving Adaptive / Responsive Signal Control Performance: Implications of Non-Invasive Detection and Legacy Timing Practices

- Lead: Northern Arizona University

- Subs:

- Portland State University (Sirisha Kothuri)

- Iowa State University (Anuj Sharma)

- Objective

- Different detection sources provide varying levels of accuracy

- The impact of less than optimal detection on traditional call and extend operation is well known

- How does sub-optimal detection impact the operation of higher level control algorithms, such as adaptive and/or traffic responsive? 


\section{Motivation}

- Desire to collect high resolution event based data from 2070 running Voyage (Northwest Signal / Peek)

- Inspiration taken from ASC/3 event based data logger worked on while at Purdue

- Desire to collect as large a sample as possible

- Need for portable event based data logger 


\section{Data Flow}

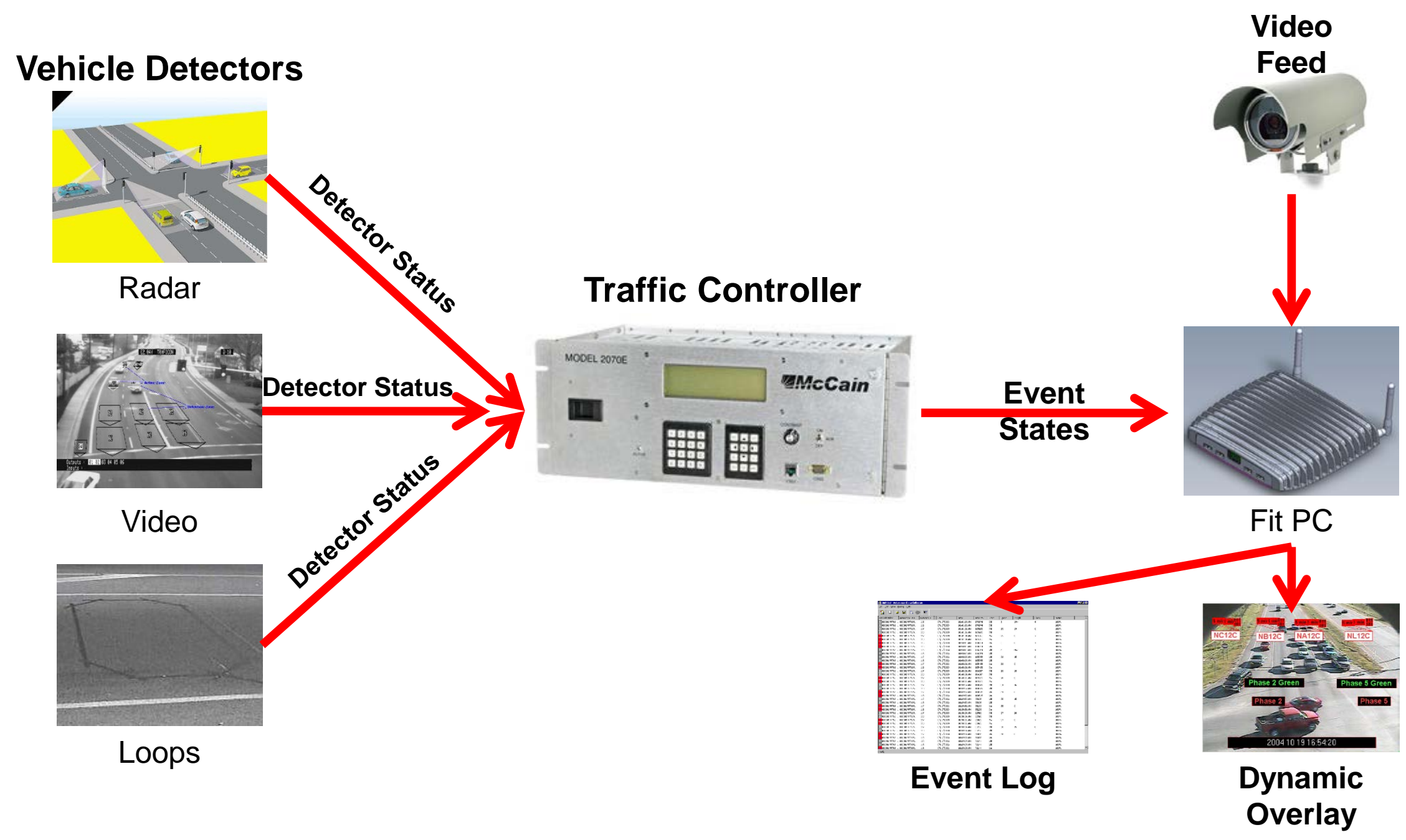




\section{Module Development}

- Northwest Signal's Testbox

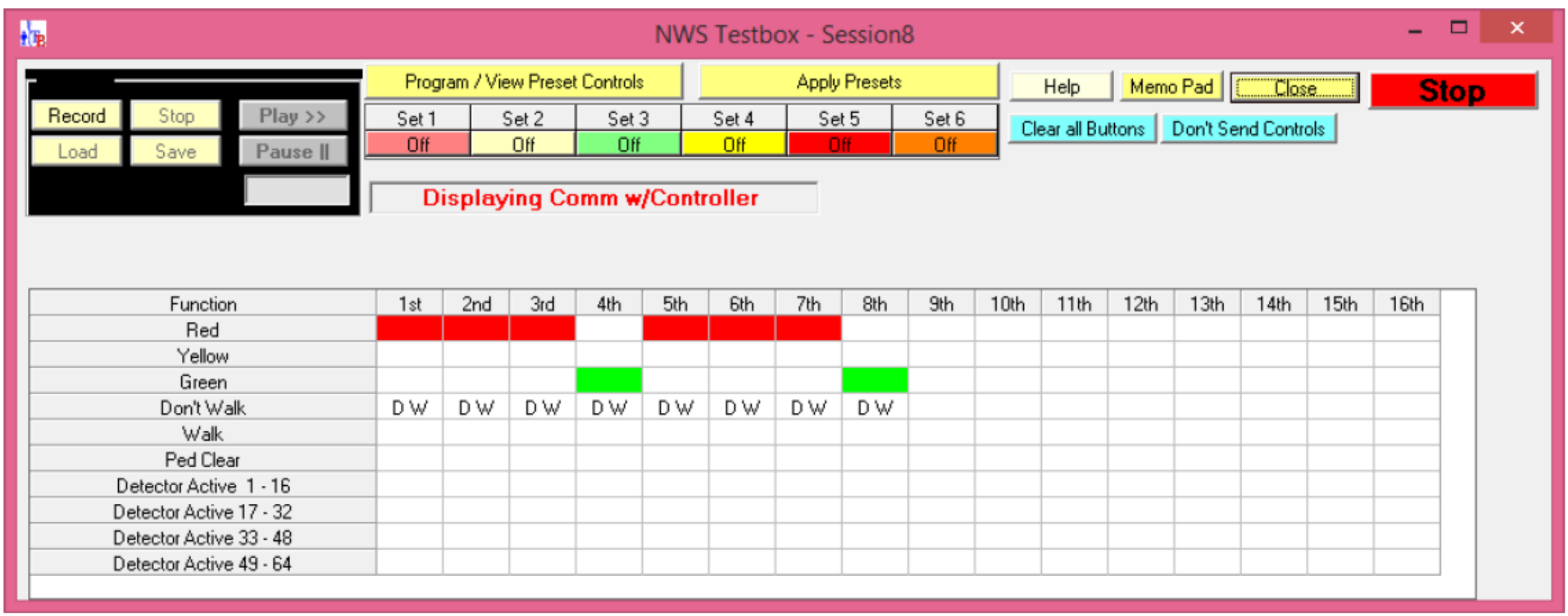




\section{Module Development}

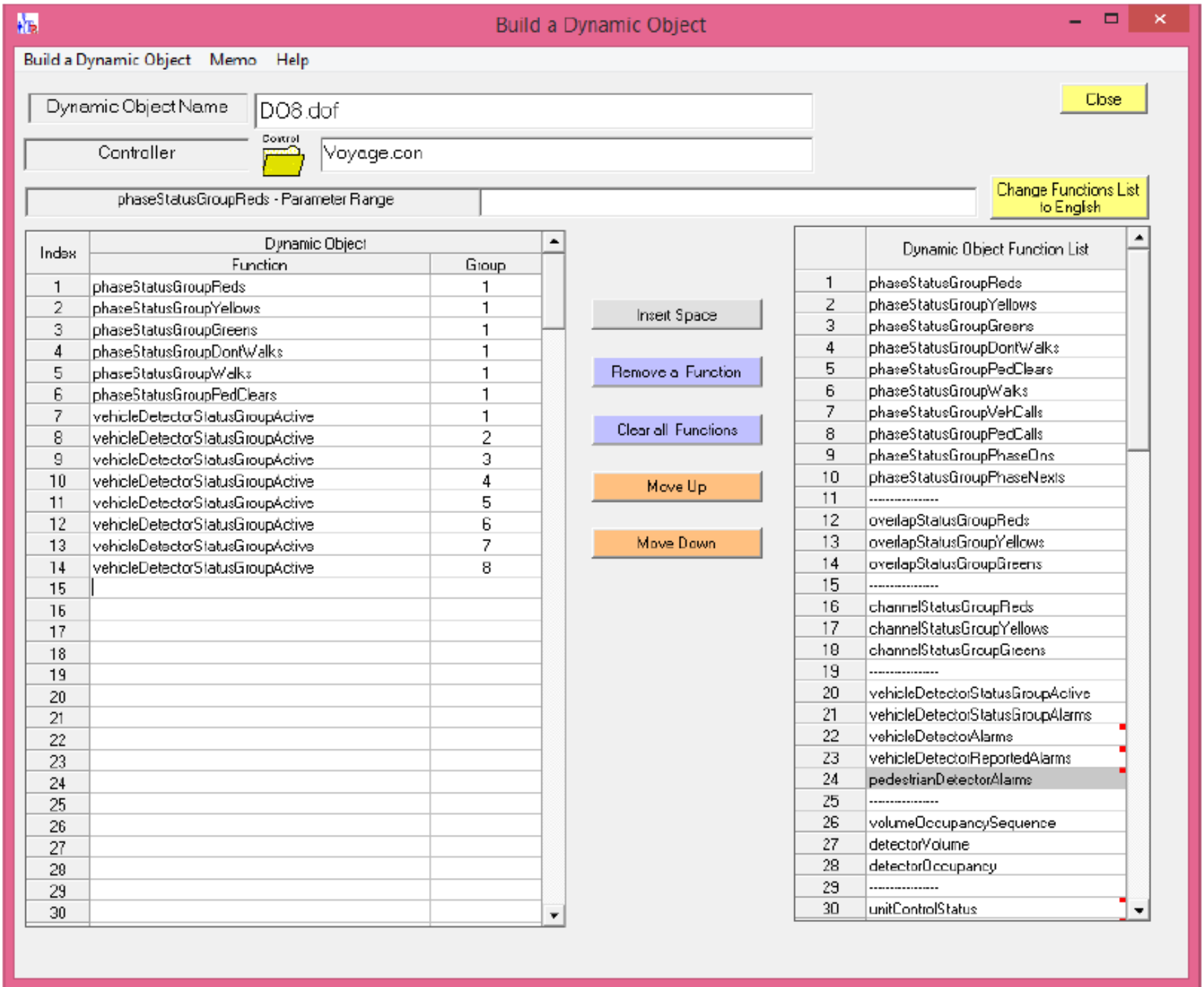




\section{Module Development}

- Data Flow Diagram

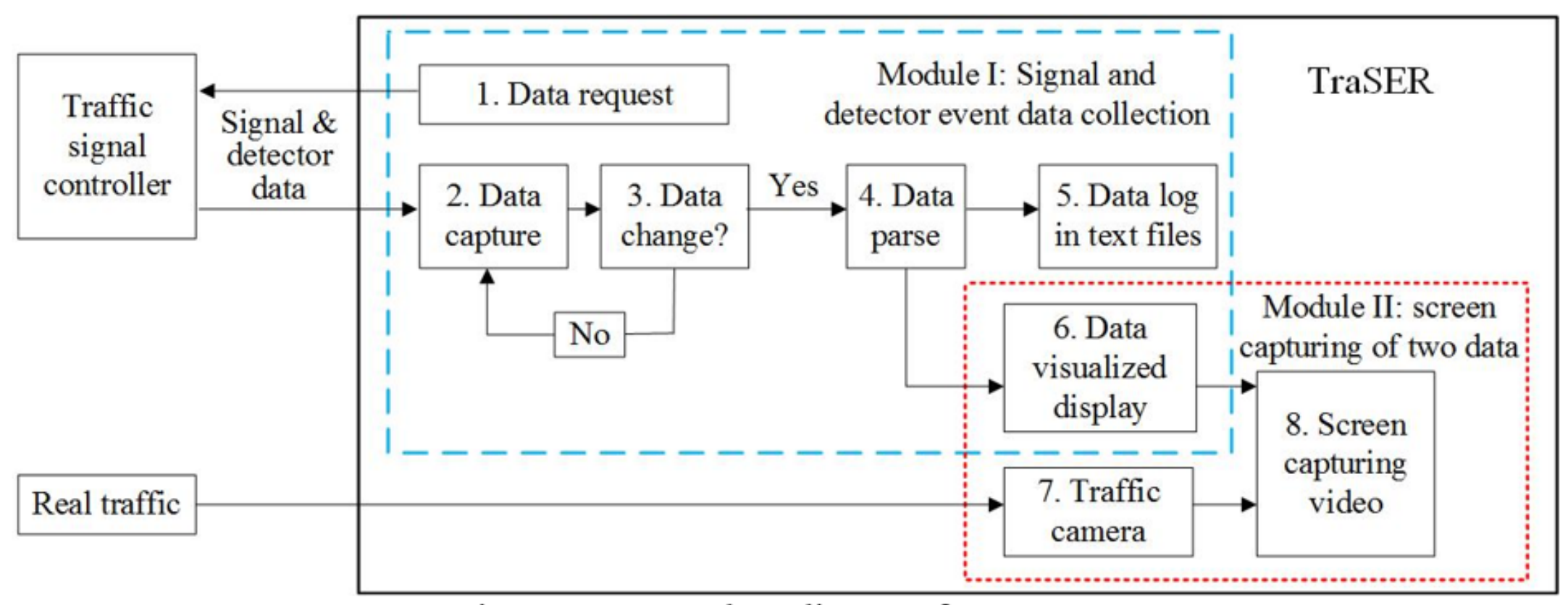




\section{Module Development}

- Visual interface

that can be

overlaid on screen

I video

- Event based data file recorded from state changes

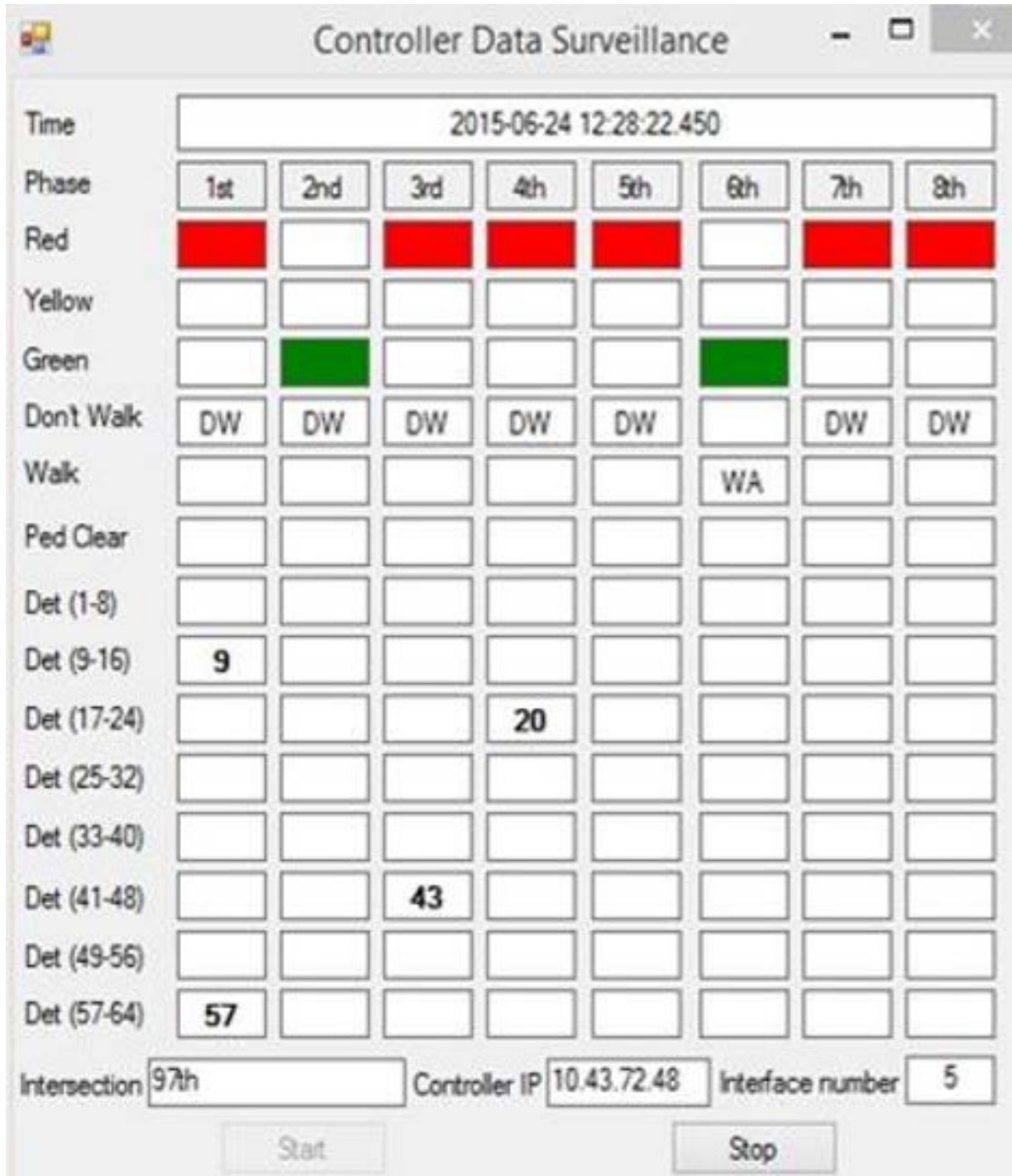




\section{Module Development}

1.. 2015070909 ChgData.txt

$1 \ldots 1, \ldots 10 \ldots 1, \ldots 20 \ldots 1, \ldots 30 \ldots 1, \ldots 40$

1 Intersection; Date; Time; Signal; Phase; Statusq

2 97th;2015-07-09;09:32:29.544;R;3;/1 q

3 97th;2015-07-09;09:32:29.544;R;4;1q

4 97th;2015-07-09;09:32:29.544;R;7;19

5 97th;2015-07-09;09:32:29.544;R;8;19

6 97th;2015-07-09;09:32:29.544;G;2;19

$797 \mathrm{th} ; 2015-07-09 ; 09: 32: 29.544 ; \mathrm{G} ; 6 ; 1 \mathrm{q}$

8 97th;2015-07-09;09:32:29.544;DW;1;19

9 97th;2015-07-09;09:32:29.544; DW;2;19

10 97th;2015-07-09;09:32:29.544;DW;3;19

11 97th;2015-07-09;09:32:29.544;DW;4;19

12 97th;2015-07-09;09:32:29.544;DW; $; 19$

13 97th;2015-07-09;09:32:29.544;DW; 6 ; 19

14 97th;2015-07-09;09:32:29.544;DW;7;19

15 97th;2015-07-09;09:32:29.544; DW;8;19

16 97th;2015-07-09;09:32:40.760;DET;9;19

17 97th;2015-07-09;09:32:41.748;DET;41;19

18 97th;2015-07-09;09:32:42.110;DET;9;0q

19 97th;2015-07-09;09:32:42.161;DET;9;19 


\section{Module Development}

B) Autoscope Video Player - 2015,06,24 12:17:15 - 10.5.0 - - 100539FF2B530236 - EB Phase 8 - 97 th @ Lawnfield - Lum mount - 2 - Terra Access Point NEMA -.

$-0$ File View Play Detector Overiay Boolomarls Window Help

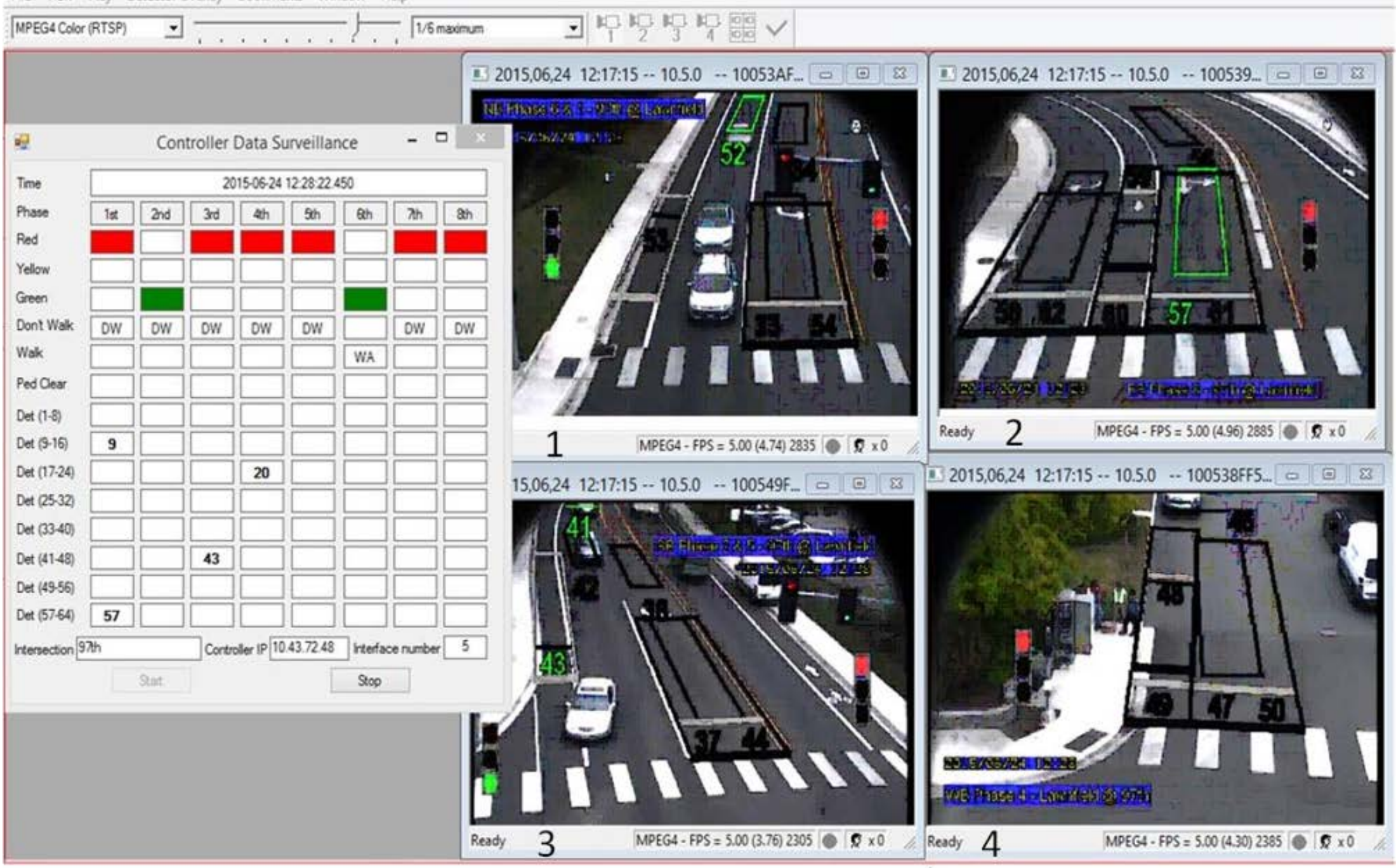




\section{Module Development}

- Use HyperCam to capture screen

- Slice video and data files into $1 \mathrm{hr}$ increments with batch operation

- Will run "indefinitely"

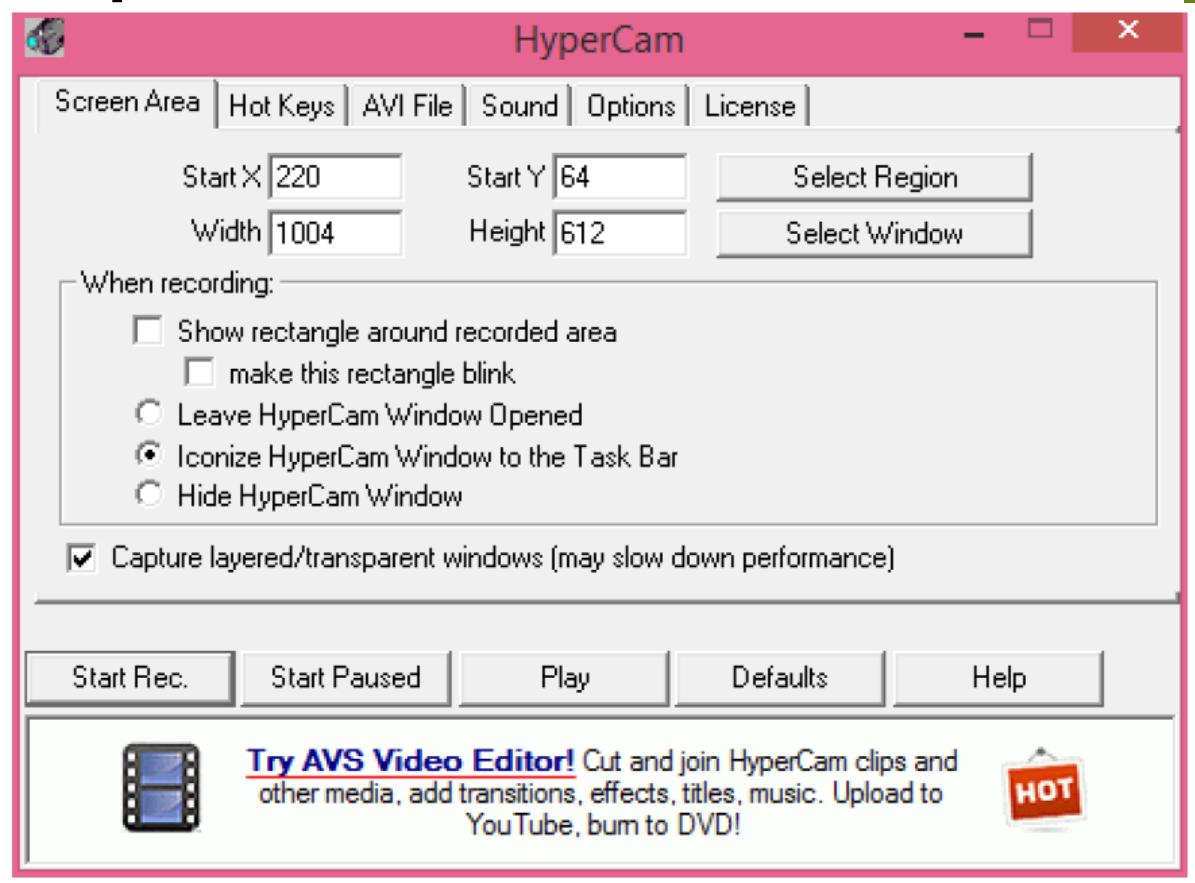




\section{Site Locations}

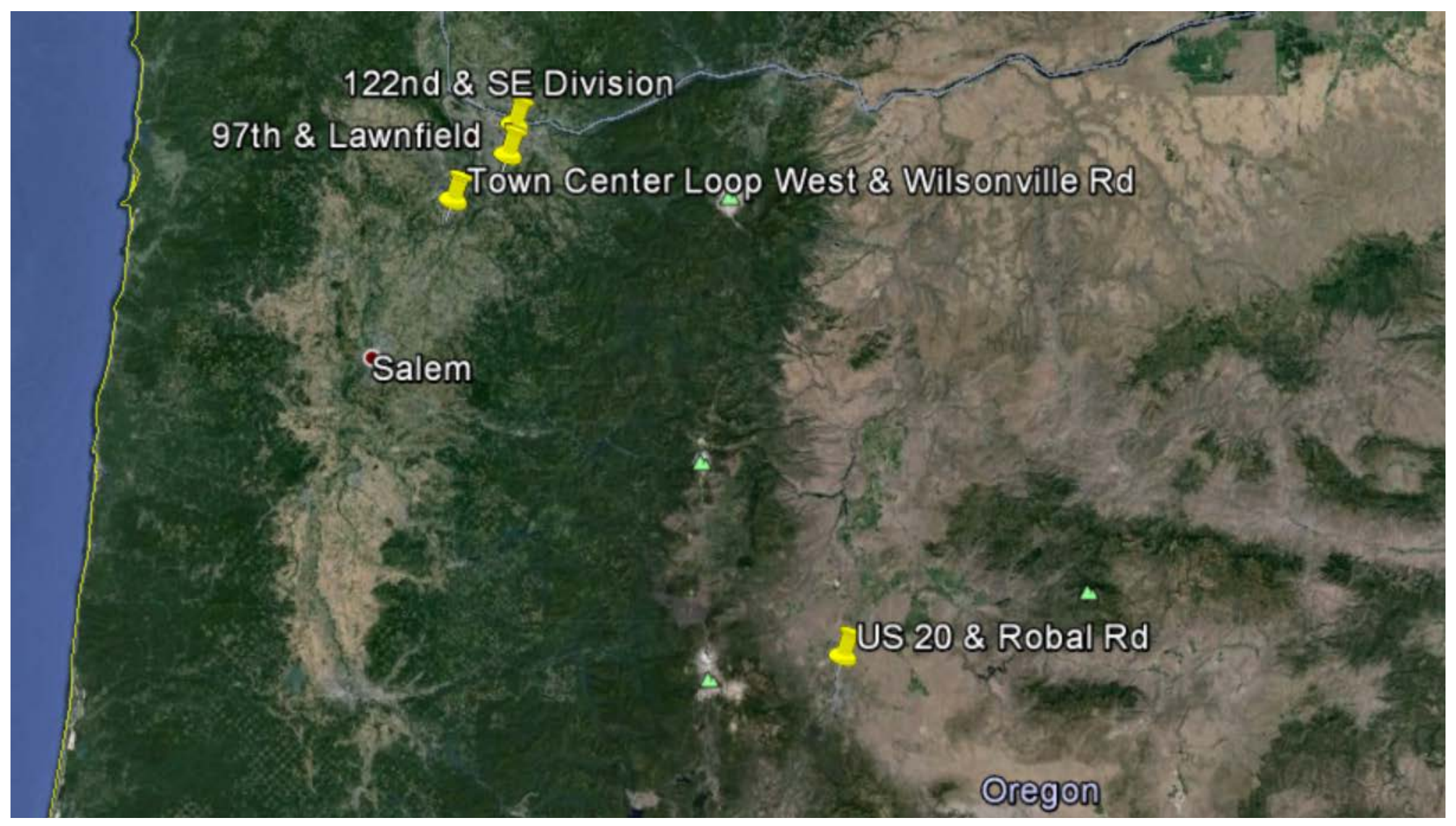




\section{Town Center Loop West \&} Wilsonville Road, Wilsonville

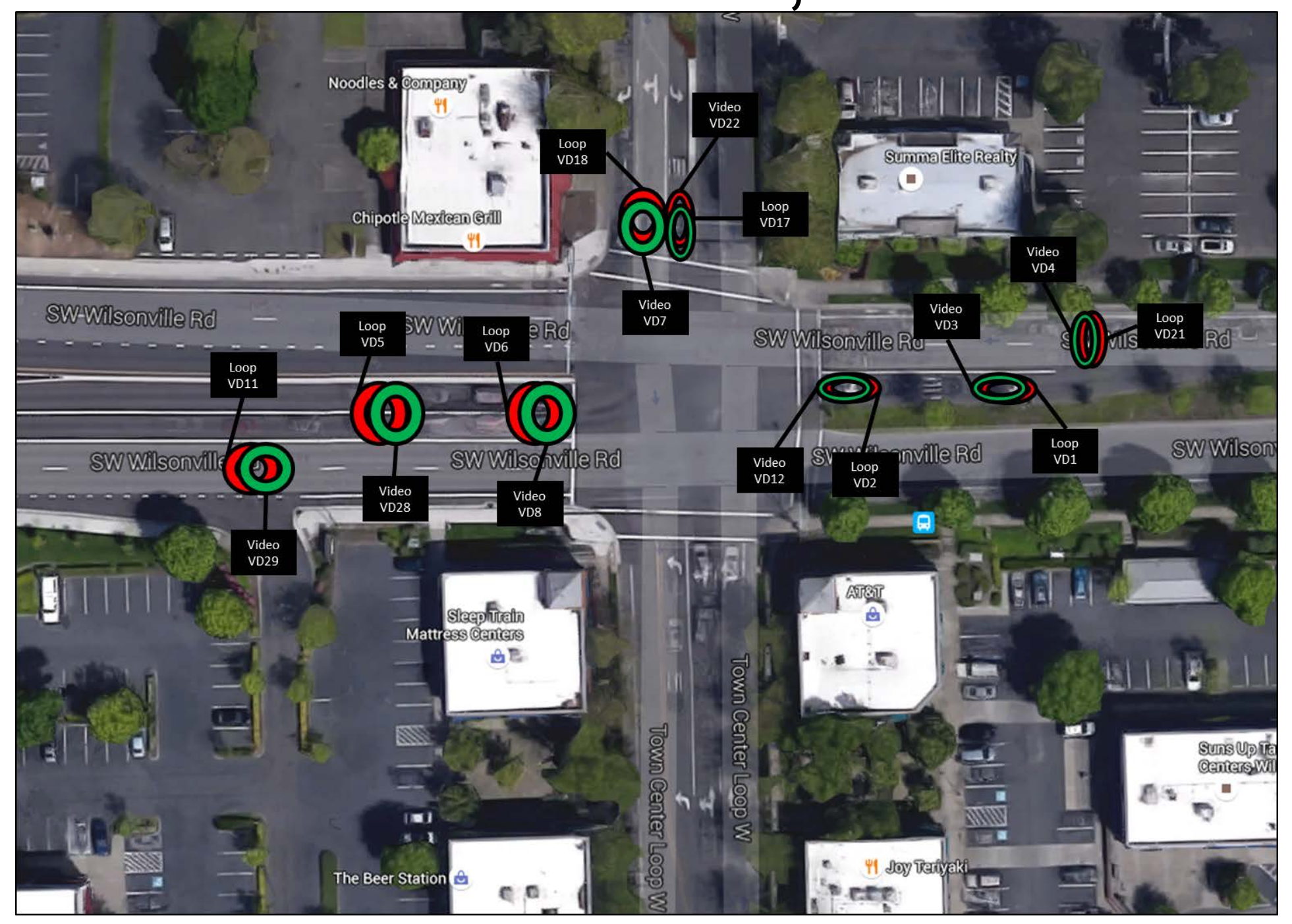




\section{$97^{\text {th }} \&$ Lawnfield, Clackamas County}

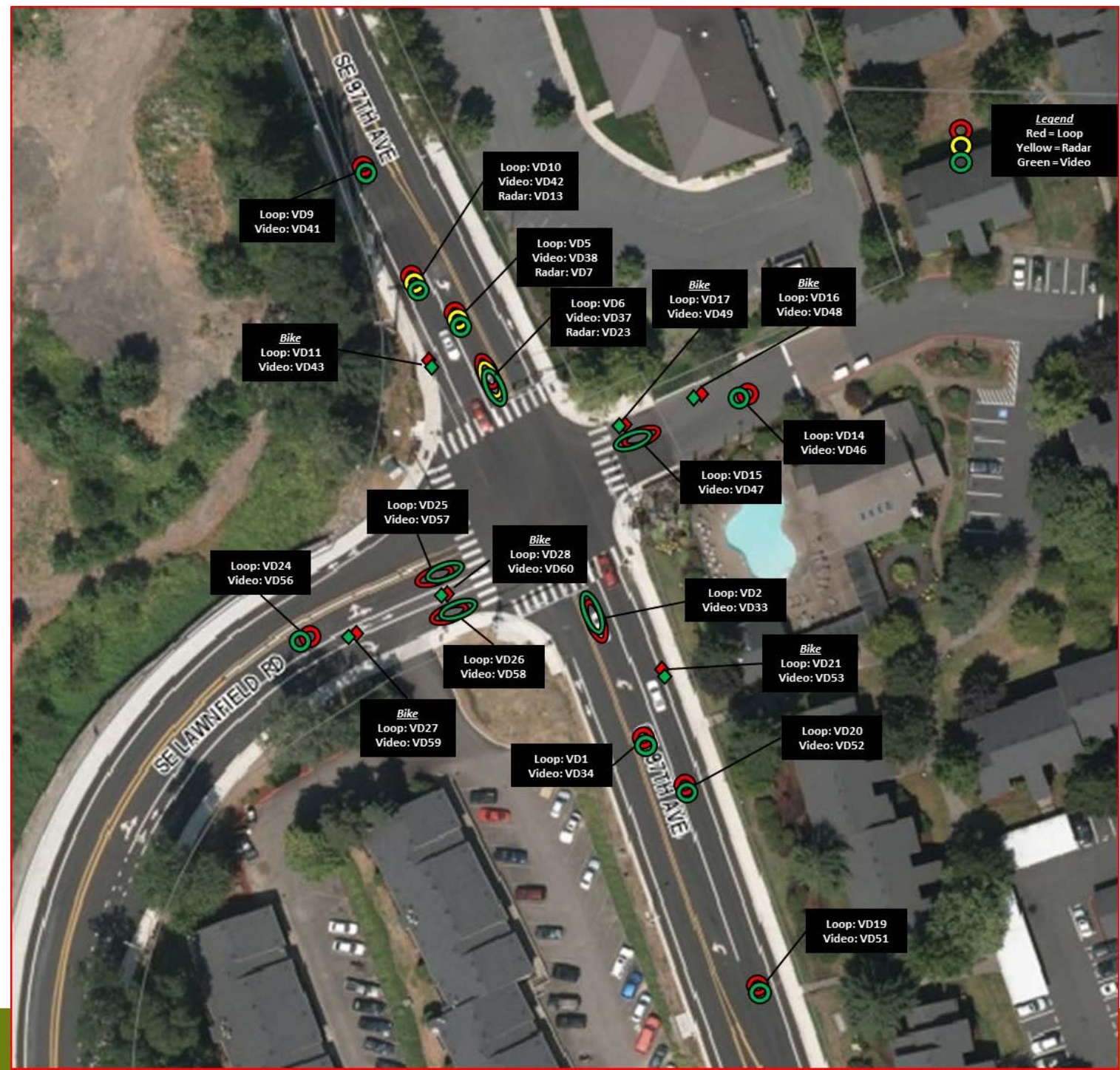




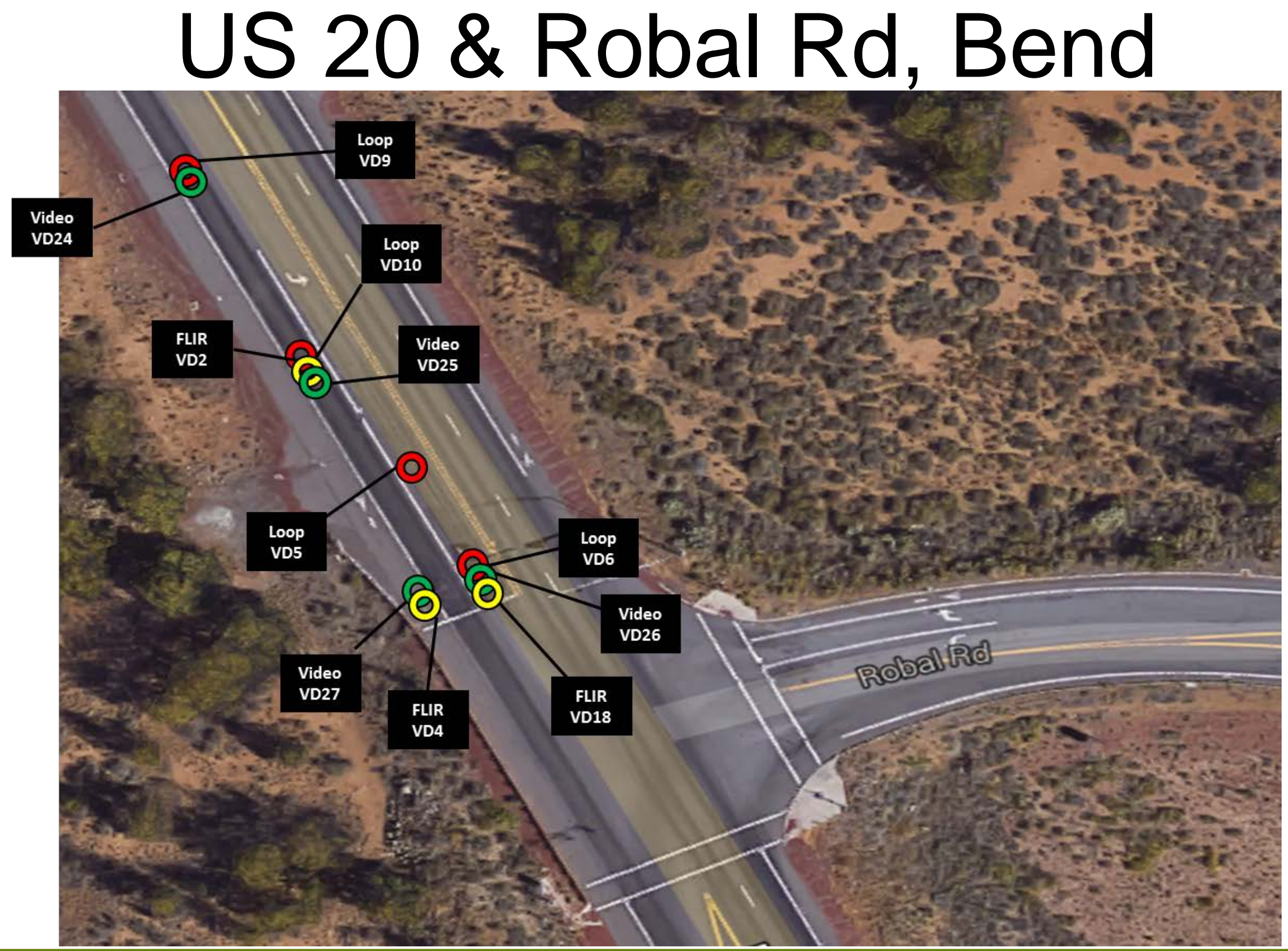


$122^{\text {nd }} \&$ SE Division, Portland
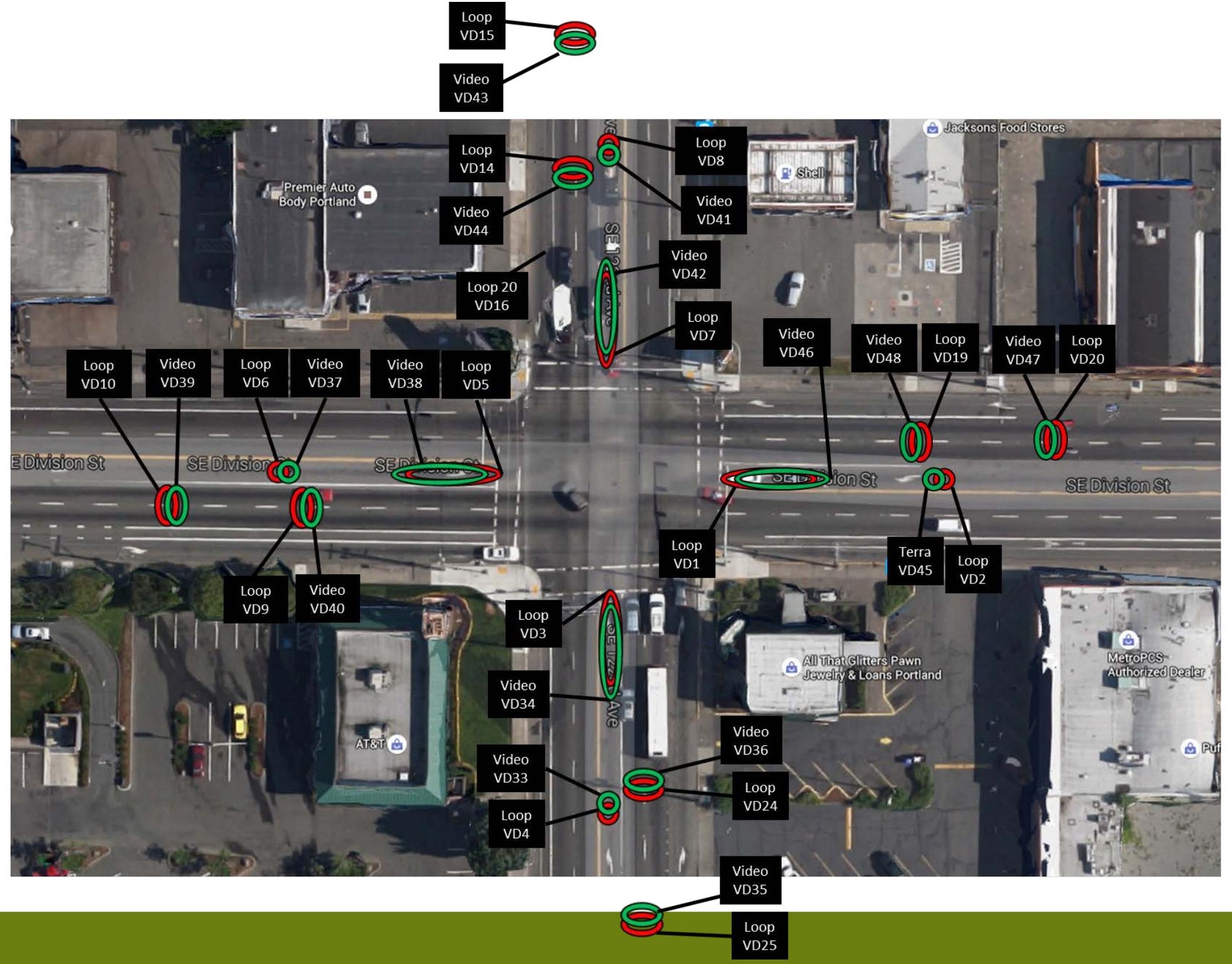


\section{Data Collection}

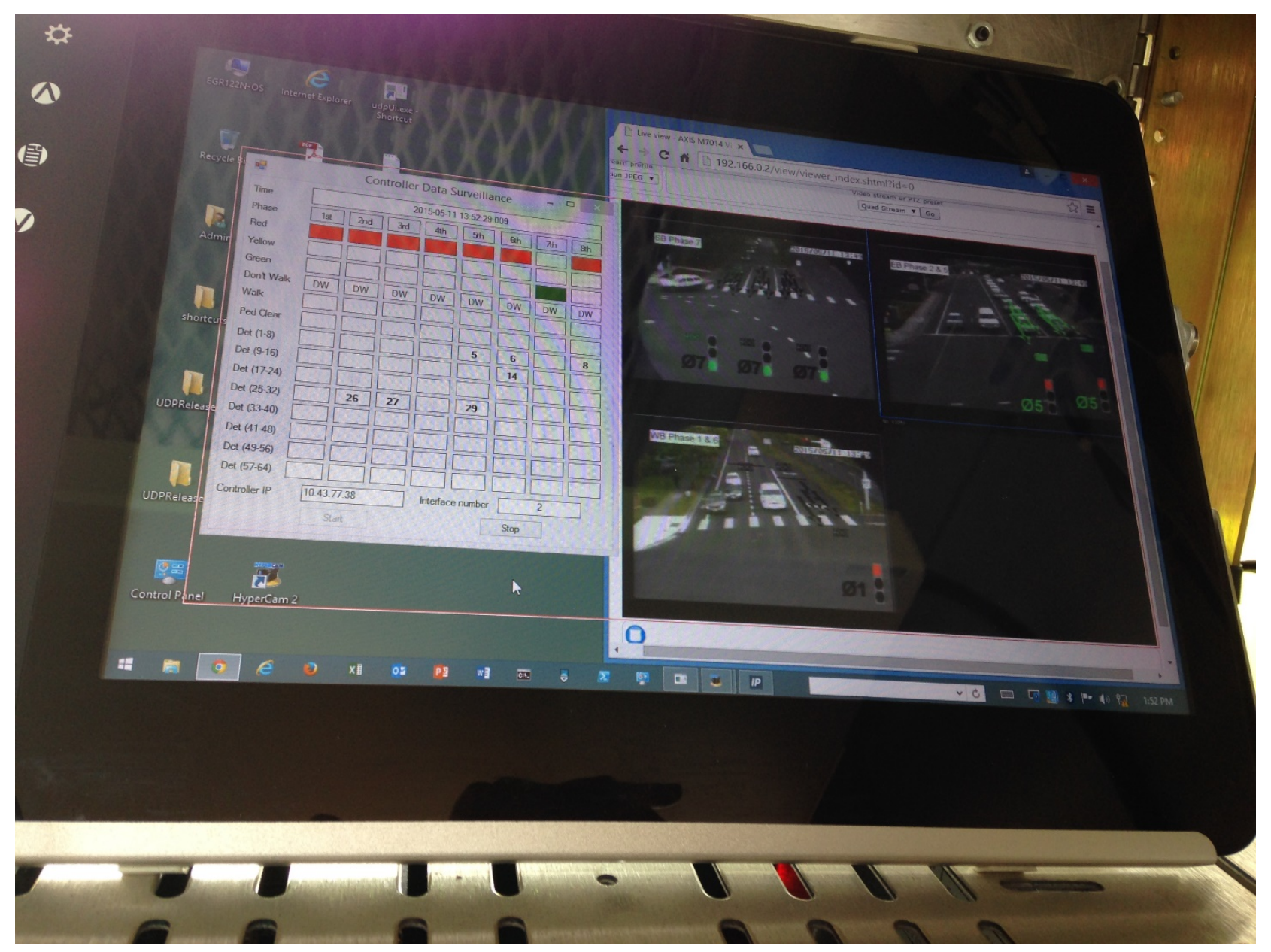




\section{Data Collection}

- Minor issues occurred at $97^{\text {th }} /$ Lawnfield \& TCLW / Wilsonville related to MS Windows pop-ups

- Major issues at Bend severely limited data collection

- $122^{\text {nd }} /$ SE Division was uneventful

\begin{tabular}{|l|l|l|}
\hline $\begin{array}{l}\text { Location } \\
\text { SW Wilsonville Rd. and Town Center }\end{array}$ & $\begin{array}{l}\text { Data Collection } \\
\text { Dates }\end{array}$ & Good Data \\
\hline Loop W & $5 / 11 / 15-6 / 18 / 15$ & 507 hrs ( 21 days $)$ \\
\hline SE $97^{\text {th }}$ Ave. and SE Lawnfield Rd. & $6 / 18 / 15-7 / 28 / 15$ & 599 hrs ( 25 days) \\
\hline US 20 and Robal Rd. & $6 / 25 / 15-11 / 6 / 15$ & 196 hrs ( 8 days $)$ \\
\hline SE Division St. and SE 122 ${ }^{\text {nd }}$ Ave. & $10 / 20 / 15-11 / 16 / 15$ & 626 hrs ( 26 days $)$ \\
\hline
\end{tabular}




\section{Preliminary Results}

- Over 5 million unique records

- Tableau used as visualization tool

tableaut:public

〈My Profile

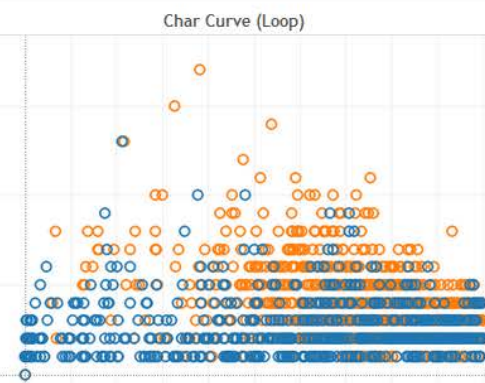

Charac Curve (Alt. Tech)
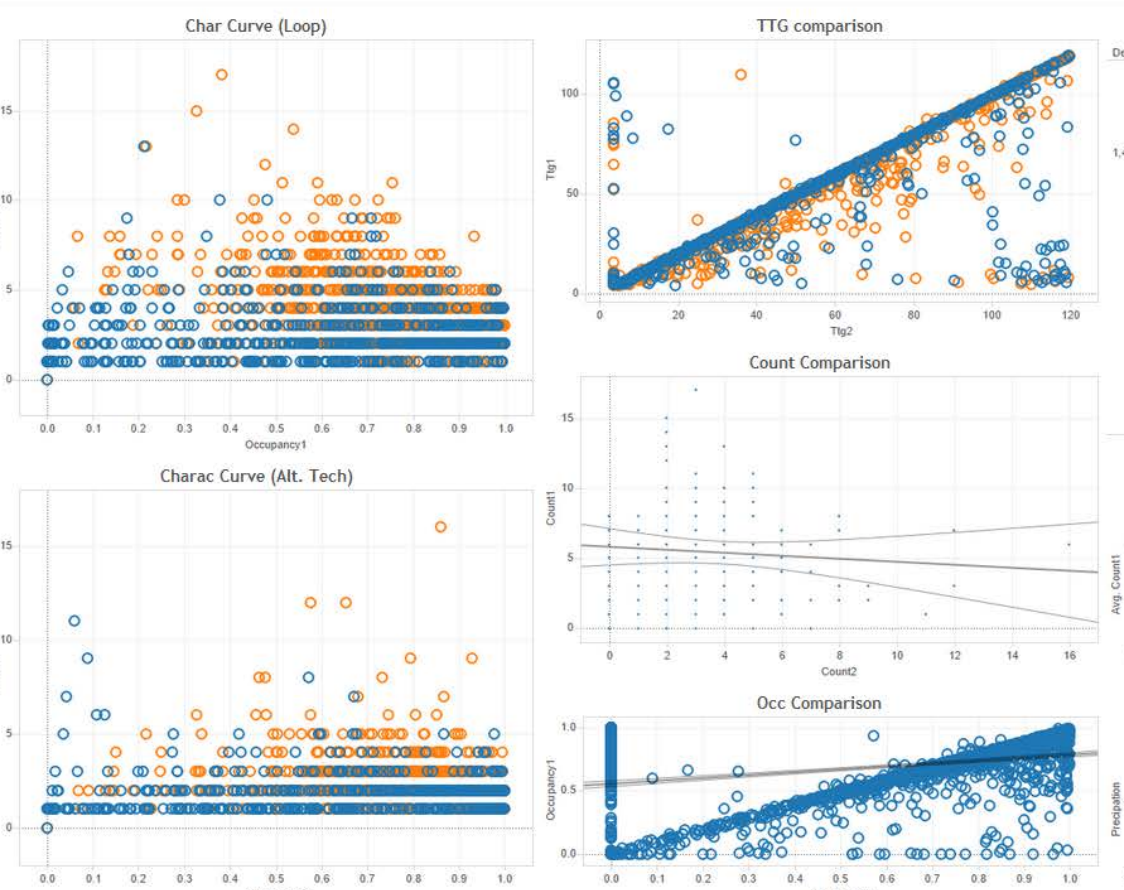

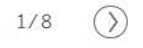

aUthors bLOG RESOURCES ACTIVITY $\bigcirc$

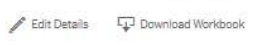

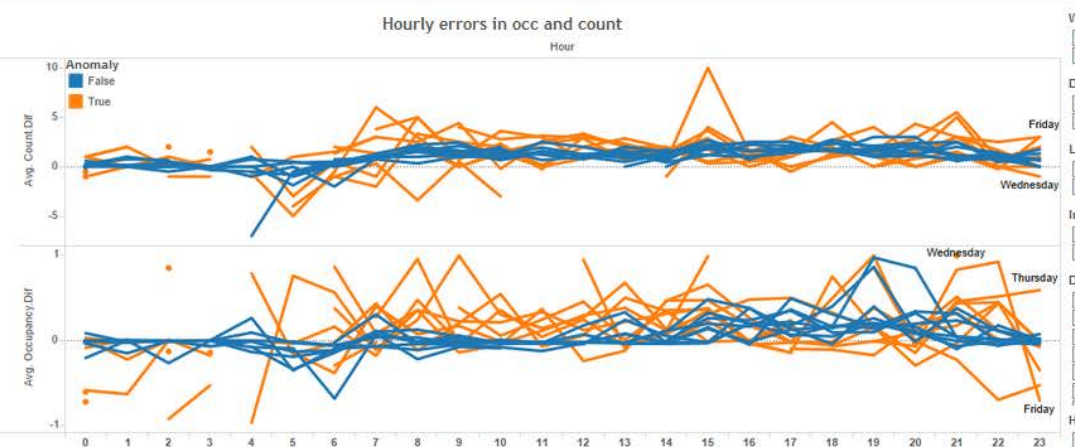

Hourly Count (Loop)
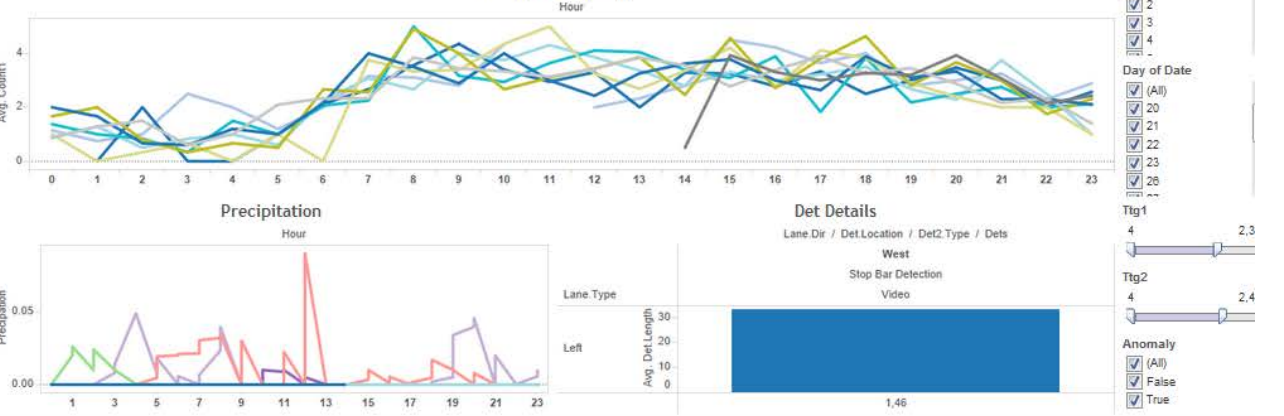


\section{Preliminary Results}

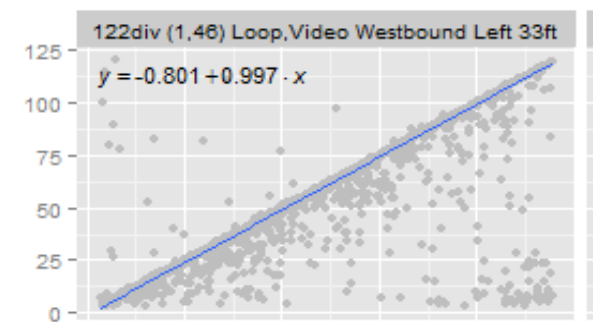

97th $(15,47)$ Loop.Video Westbound Left/Thru/Right

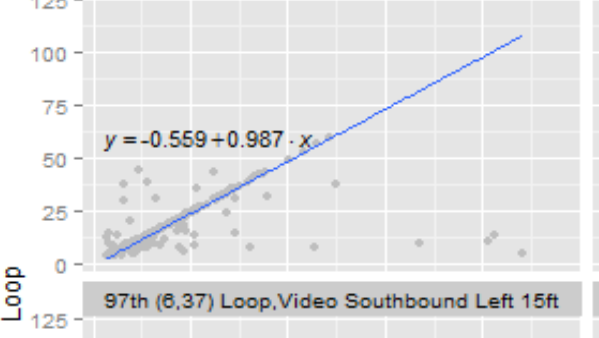

$y=-0.537+0.974 \cdot x$

(3)

wilson $(2,12)$ Loop.Video Westbound Left $15 \mathrm{ft}$ $y=-1.13+1 \cdot x$

$100-$

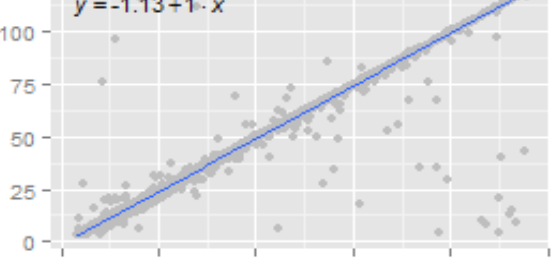

First time to gap out of stop bar detectors

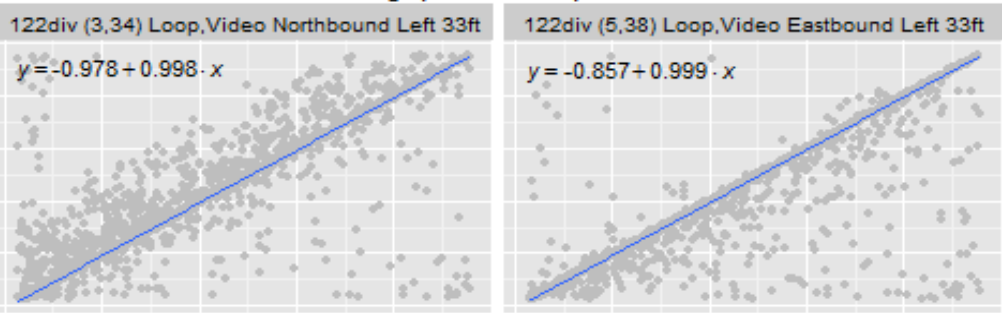

97th $(25,57)$ Loop, Video Esstbound Left/Thru 15 97 th $(2,33)$ Loop,Video Northbound Left $15 \mathrm{ft}$ $y=-0.495+0.993 \cdot x$

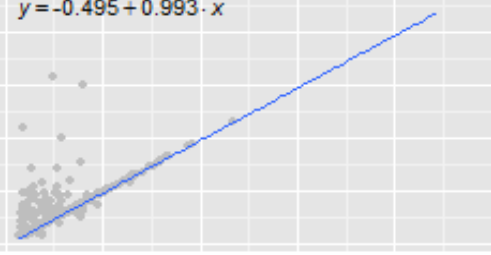

bend $(6,26)$ Loop.Video Southbound Left $15 \mathrm{ft}$ $\dot{y}=-0.542+1 \cdot x$

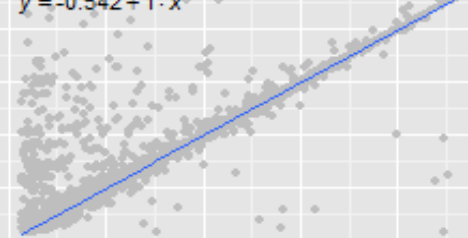

wilson $(6,8)$ Loop.Video Esstbound Left $15 \mathrm{ft}$ $y=-0.681-1 \cdot x$
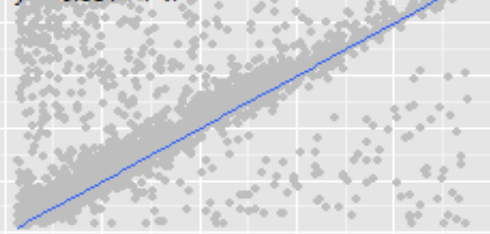

122div (7.42) Loop, Video Southbound Left 33ft

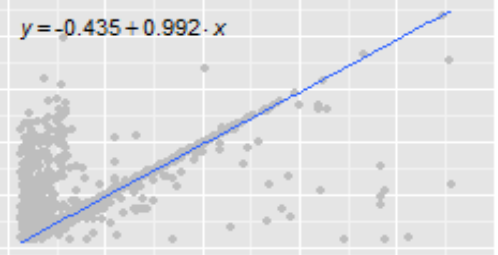

wilson $(17,22)$ Loop.Video Southbound Left $8 \mathrm{ft}$ $y=-1.53+1 \cdot x$

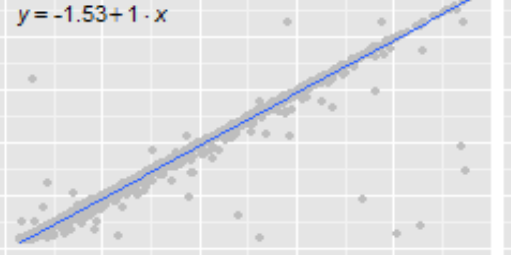
$y=-0.635+0.997 \cdot x$

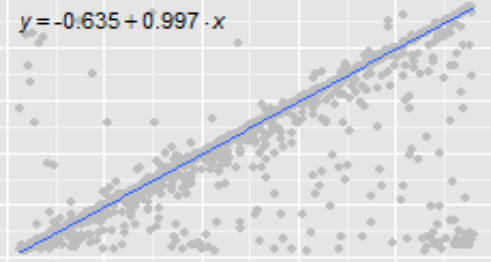

97th $(26,58)$ Loop.Video Esstbound Right $15 \mathrm{ft}$

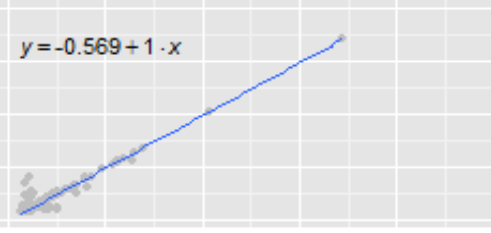

wilson $(18,7)$ Loop.Video Southbound Thru $15 \mathrm{ft}$

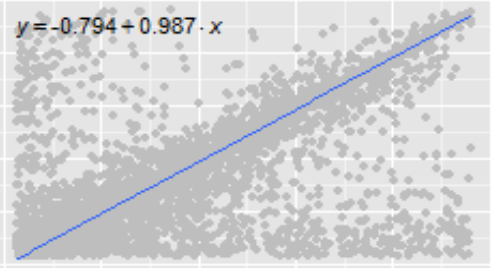




\section{Lessons Learned}

- Use Linux (yeah, we probably knew this before we started)

- IT policies make it challenging for an external partner to monitor data collection

- Data lost due to site visit gaps

- Support from project partners is critical

- ODOT

- Clackamas County

- Portland Bureau of Transportation 


\section{Lessons Learned}

- Support from vendors is also critical

- Northwest Signal / Peek

- Detection vendors / manufacturers

- While data collection module does not need to be on site, much bandwidth needed

- Processing power can be an issue 


\section{Next steps}

- Very promising for data collection under Voyage

- Ability to monitor virtually anything in controller (Dynamic Object set)

- Future of Voyage in question, however

- Scalable to other platforms, however detector status by channel must be reported 


\section{Acknowledgements}

- Oregon Department of Transportation

- Jon Lazarus, Boettcher, Dave Hirsch and SPR 781 TAC

- Dan Carson and Jon Meusch, formerly of Northwest Signal / Peek

- Clackamas County

- Bikram Raghubansh

- Portland Bureau of Transportation

- Paul Zebell 


\section{Questions?}

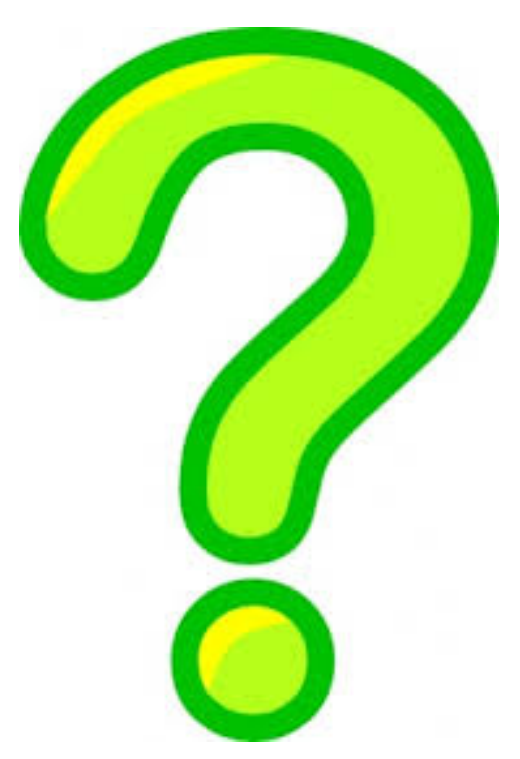




\section{Thank you!}

